\title{
The tetrahydrobiopterin pathway: a novel target for the treatment of depression
}

\section{"...there is a clear justification for studying the $\mathrm{BH}_{4}$ pathway to improve treatment strategies for depression."}

\section{KEYWORDS: antidepressants $\approx \mathrm{BH}_{4} \approx$ depression $\approx$ pharmacogenetics - tetrahydrobiopterin}

Existing treatments for depression usually take several weeks to achieve their antidepressive effects, and a large number of patients do not show a significant improvement in their symptoms or are treatment refractory, even after long-term drug administration. As a result, there is an urgent need for therapeutics with improved efficacy that can exert their effects within hours or days of administration. The pteridine tetrahydrobiopterin $\left(\mathrm{BH}_{4}\right)$ is an essential cofactor for the production of many neurotransmitters including serotonin (5-HT). Given the crucial role that the $\mathrm{BH}_{4}$ pathway has in neurotransmitter production and observations that this pathway is central to the pathogenesis of several diseases including depression, there is a clear justification for studying the $\mathrm{BH}_{4}$ pathway to improve treatment strategies for depression.

Depression is one of the most common psychiatric disorders with far-reaching social and economic costs. It has a lifetime prevalence of nearly $20 \%$ in industrialized countries and accounts for $10 \%$ of all productive years lost throughout the world [1]. The WHO predicts that by the year 2020, depression will be the greatest burden of ill health to people in the developing world, second only to heart disease as the largest cause of death and disability, with $10-15 \%$ of depressed individuals committing suicide.

The major challenge facing clinicians is predicting who will develop depression and identifying the most effective treatments for those individuals. Antidepressant therapy can dramatically reduce suicide and hospitalization rates [2]. However, as a general rule, each antidepressant is only effective for approximately $50 \%$ of patients, and $5-10 \%$ of patients are treatment refractory and do not respond to any of the currently available antidepressants [3]. Furthermore, it takes several weeks for a therapeutic response or lack of response to become apparent, and during this period, patients suffer ongoing morbidity, adverse effects of drugs and increased risk of suicide. Therefore, choosing the right antidepressant for each patient is critical and a significant challenge in psychiatry.

According to the monoamine theory of depression, the therapeutic effects of antidepressants are primarily owing to the ability of these drugs to block the reuptake of the monoamines 5-HT and noradrenaline. Selective serotonin-reuptake inhibitors and noradrenaline reuptake inhibitors specifically prevent the reuptake of 5-HT and noradrenaline, respectively (thereby increasing the level of 5-HT or noradrenaline in synapses of the brain). Although these drugs are clearly effective in treating depression, the monoaminergic theory still leaves important unanswered questions. In particular, whilst antidepressants reach therapeutic concentrations in the blood within days and begin to alter neurotransmitter activity, changes in mood take weeks to appear. One explanation for this delay is that slow adaptive or neuroplastic changes to neurotransmitter receptors and associated signalling pathways are required for therapeutic benefit to occur [4]. Another possible explanation is that antidepressants may derive their effects by promoting neurogenesis in the hippocampus [4]. Alternatively, antidepressants, rather than regulating the release of neurotransmitters, may exert their effects through pathways that regulate the synthesis of neurotransmitters. One such pathway is the pteridine pathway, which produces a chemical called $\mathrm{BH}_{4}$, which plays an essential role in the production of neurotransmitters.

$\mathrm{BH}_{4}$ is the essential cofactor for the conversion of phenylalanine to tyrosine, the generation of nitric oxide, as well as the hydroxylation of tyrosine and tryptophan, the rate-limiting steps in the formation of dopamine, noradrenaline

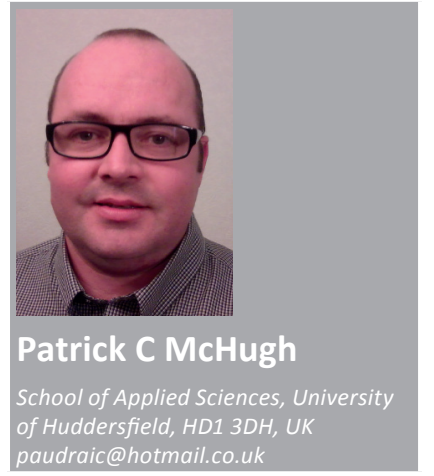

future porate $\mathrm{fSg}$ 
and 5-HT [5]. The initial and rate-limiting step in the de novo synthesis of $\mathrm{BH}_{4}$ is the conversion of GTP to 7,8-dihydroneopterin triphosphate by way of the enzyme GTPCH; this is followed by a series of conversions by two other enzymes, SPR and PTPS leading to the production of $\mathrm{BH}_{4} \cdot \mathrm{BH}_{4}$ can also be regenerated and salvaged through a series of reactions separate to the de novo pathway. The pathway is regulated by $\mathrm{GTPCH}$ feedback regulator (GCHFR), which mediates feedback inhibition of GTPCH activity via $\mathrm{BH}_{4}$; the inhibition by $\mathrm{BH}_{4}$ is reversed by phenylalanine. Over the past 30 years the $\mathrm{BH}_{4}$ pathway has been linked to many conditions, including mood disorders, autism, Alzheimer's disease, Parkinson's disease (PD) and neuropathic pain [5,6].

\section{"Despite the moderate efficacy of currently available antidepressant therapies, there is a clear need for improved strategies for the treatment of depression..."}

Pharmacogenomic studies investigating moodregulating drugs, including antidepressants, have demonstrated altered gene and protein expression in the $\mathrm{BH}_{4}$ pathway [5,7]. Other studies have demonstrated that variants of two genes in the $\mathrm{BH}_{4}$ pathway, GCHFR and $S P R$, are associated with clinical selective serotoninreuptake inhibitor response $[8,9]$. In relation to PD, antidepressants have shown to have similar properties to nitric oxide synthase inhibitors, which have antidepressant-like effects and are neuroprotective in PD [10]. Furthermore, SPR gene variants have been associated with mood disorders and PD [9,11]. Intriguingly, there is evidence to suggest that depression actually precedes and is independent of early PD symptoms [12]. Several studies have investigated the efficacy of antidepressants in PD patients with depression with some encouraging findings [13]. In fact, depressed patients with PD, treated with antidepressants, demonstrated improved cognitive functioning [14], which further highlights the importance of this pathway in several diseases and their treatments.

There is a wealth of clinical evidence to suggest that depression occurs with high frequency among patients suffering with chronic or neuropathic pain. Approximately $30 \%$ of patients with persistent pain conditions suffer from clinical depression and $75 \%$ of patients with clinical depression present with physical symptoms of pain $[15,16]$. There is evidence that depression and pain have overlapping neurobiologic mechanisms and that their neurochemistry is inextricably linked [17]. Antidepressant drugs have been widely used for many years to treat neuropathic pain, and as in the treatment of depression, there has been poor efficacy and thus a clear need for improved therapeutics $[18,19]$.

A recent link between pain and the $\mathrm{BH}_{4}$ pathway has been observed [6]. The gene for GTPCH $(G C H I)$, the rate-limiting enzyme in the synthesis of $\mathrm{BH}_{4}$, and SPR have been found to be dysregulated in preclinical pain models and levels of $\mathrm{BH}_{4}$ have been shown to be critical in neuropathic and inflammatory pain [20]. Furthermore, a specific GCHI haplotype has been found to be protective against pain sensitivity, owing to the associated reduction of $\mathrm{BH}_{4}$ production [20]. This link between the $\mathrm{BH}_{4}$ pathway and pain has led to some exciting new research into the development of analgesic therapies that target this pathway [6,21]. Amongst the compounds that have been identified, the two most promising candidates include: the GTPCH inhibitor, 2,4-diamino-6-hydroxypyrimidine and the SPR inhibitor, $N$-acetylserotonin [21]. In fact, $N$-acetylserotonin has been observed to have antidepressant activity in animal models of depression [22].

Despite the moderate efficacy of currently available antidepressant therapies, there is a clear need for improved strategies for the treatment of depression, as well as diseases that rely on antidepressant therapies. Studies investigating the pathobiology and treatment of $\mathrm{PD}$, pain and mood disorders have highlighted the $\mathrm{BH}_{4}$ pathway as a novel target for the development of new treatments and personalized antidepressant therapies. Although the potential of this pathway requires thorough examination before it can be translated into therapeutic reality, it clearly provides an exciting area of research that plausibly holds promise for new therapies for these devastating diseases.

\section{Acknowledgements}

The author would like to thank the editors of Pharmacogenomics.

Financial \& competing interests disclosure The author has no relevant affiliations or financial involvement with any organization or entity with a financial interest in or financial conflict with the subject matter or materials discussed in the manuscript. This includes employment, consultancies, honoraria, stock ownership or options, expert testimony, grants or patents received or pending, or royalties.

No writing assistance was utilized in the production of this manuscript. 


\section{References}

1 Trivedi JK, Sharma S, Tandon R. Depression in general clinical practice. J. Indian Med. Assoc. 102, 557-558 (2004).

2 Rutz W, Walinder J, Eberhard G et al. For the Swedish PTD Committee. Prevention of suicide. Lancet 340, 1233-1234 (1992).

3 Frazer A. Antidepressants. J. Clin. Psychiatry 58(Suppl. 6), 9-25 (1997).

4 Nemeroff CB, Owens MJ. Treatment of mood disorders. Nat. Neurosci. 5(Suppl.), 1068-1070 (2002).

5 McHugh PC. Tetrahydrobiopterin pathway may provide novel molecular targets for acute and long term efficacy of mood-regulating drugs. Curr. Pharmacogenomics Person. Med. 8, 174-181 (2010).

6 Latremoliere A, Costigan M. GCH1, $\mathrm{BH}_{4}$ and pain. Curr. Pharm. Biotechnol. 12(10), 1728-1741 (2011).

7 McHugh PC, Rogers GR, Loudon B, Glubb DM, Joyce PR, Kennedy MA. Proteomic analysis of embryonic stem cellderived neural cells exposed to the antidepressant paroxetine. J. Neurosci. Res. 86, 306-316 (2008).

8 McHugh PC, Joyce PR, Deng X, Kennedy MA. A polymorphism of the GTP-cyclohydrolase I feedback regulator gene alters transcriptional activity and may affect response to SSRI antidepressants. Pharmacogenomics J. 11, 207-213 (2011).
9 McHugh PC, Joyce PR, Kennedy MR. Polymorphisms of sepiapterin reductase gene alter promoter activity and may influence risk of bipolar disorder. Pharmacogenet. Genomics 19, 330-337 (2009).

10 Dunnett SB, Bjorklund A. Prospects for new restorative and neuroprotective treatments in Parkinson's disease. Nature 399, A32-A39 (1999).

11 Karamohamed S, DeStefano AL, Wilk JB et al. A haplotype at the PARK3 locus influences onset age for Parkinson's disease: the GenePD study. Neurology 61, 1557-1561 (2003).

12 Martinez-Martin P, Damian J. Parkinson disease: depression and anxiety in Parkinson disease. Nat. Rev. Neurol. 6, 243-245 (2010).

13 Devos D, Dujardin K, Poirot I et al. Comparison of desipramine and citalopram treatments for depression in Parkinson's disease: a double-blind, randomized, placebocontrolled study. Mov. Disord. 23, 850-857 (2008).

14 Dobkin RD, Menza M, Bienfait KL et al. The impact of antidepressant treatment on cognitive functioning in depressed patients with Parkinson's disease. J. Neuropsychiatry Clin. Neurosci. 22, 188-195 (2010).

15 Angst F, Verra ML, Lehmann S, Aeschlimann A, Angst J. Refined insights into the pain-depression association in chronic pain patients. Clin. J. Pain 24, 808-816 (2008).
16 Bair MJ, Robinson RL, Katon W, Kroenke K. Depression and pain comorbidity: a literature review. Arch. Intern. Med. 163, 2433-2445 (2003).

17 Rome HP Jr, Rome JD. Limbically augmented pain syndrome (LAPS): kindling, corticolimbic sensitization, and the convergence of affective and sensory symptoms in chronic pain disorders. Pain Med. 1, 7-23 (2000).

18 Saarto T, Wiffen PJ. Antidepressants for neuropathic pain. Cochrane Database Syst. Rev. 3, CD005454 (2007).

19 Verdu B, Decosterd I, Buclin T, Stiefel F, Berney A. Antidepressants for the treatment of chronic pain. Drugs 68, 2611-2632 (2008).

20 Tegeder I, Costigan M, Griffin RS et al. GTP cyclohydrolase and tetrahydrobiopterin regulate pain sensitivity and persistence. Nat. Med. 12, 1269-1277 (2006).

21 Naylor AM, Pojasek KR, Hopkins AL, Blagg J. The tetrahydrobiopterin pathway and pain. Curr. Opin. Investig. Drugs 11, 19-30 (2011).

22 Jang SW, Liu X, Pradoldej S et al. $N$-acetylserotonin activates $\operatorname{TrkB}$ receptor in a circadian rhythm. Proc. Natl Acad. Sci. USA 107, 3876-3881 (2009). 